\title{
Frecuencia de micronúcleos en eritrocitos con coloración secuencial Giemsa-naranja de acridina en el gato doméstico (Felis domesticus)
}

\author{
FREQUENCY OF MICRONUCLEI IN ERYTHROCYTES WITH SEQUENTIAL GIEMSA-ORANGE \\ ACRIDINE STAINING IN THE DOMESTIC CAT (Felis domesticus)
}

Arnoldo A.M. Quero ${ }^{1,2}$, Irma Muñoz ${ }^{1}$, Daniela M. Ferré ${ }^{1,2}$, Nora B.M. Gorla ${ }^{1,2}$

\section{Resumen}

Los felinos domésticos comparten el ambiente doméstico con el hombre y tienen condiciones que los hacen especialmente sensibles a compuestos genotóxicos, por lo que son buenos candidatos como bioindicadores en el biomonitoreo de ambientes domésticos. El objetivo del estudio fue aplicar el ensayo de micronúcleos en eritrocitos de gatos domésticos (Felis domesticus) y evaluarlos mediante las coloraciones de Giemsa y naranja de acridina (NA) fluorescente realizada en forma secuencial, para identificar la más adecuada para esta especie. Se aplicó el ensayo de micronúcleos en eritrocitos de sangre periférica de gatos domésticos adultos, con y sin signos de enfermedad (total=24). Las frecuencias de micronúcleos detectadas con NA fueron 2 a 10 veces superiores que las registradas con Giemsa $(\mathrm{p}<0.0001)$, sin diferencias por factor edad o estado sanitario. Se hipotetiza que la coloración con NA permitió la detección de micronúcleos de menor tamaño que el análisis en Giemsa. La frecuencia de micronúcleos observada propone la utilización del gato doméstico para realizar biomonitoreos sobre el efecto de genotóxicos ambientales.

Palabras clave: naranja de acridina; eritrocitos micronucleados; Felis domesticus; mascotas

\section{Abstract}

Domestic felines share the domestic environment with humans and have conditions that make them especially sensitive to genotoxic compounds, they are good candidates to be used as bioindicators in the biomonitoring of domestic environments. The objective

\footnotetext{
${ }^{1}$ Laboratorio de Genética, Ambiente y Reproducción, Universidad Juan Agustín Maza, Mendoza, Argentina

${ }^{2}$ Consejo Nacional de Investigaciones Cientificas y Técnicas - CONICET, Argentina

${ }^{3}$ E-mail: noragorla@gmail.com
}

Recibido: 9 de noviembre de 2017

Aceptado para publicación: 12 de abril de 2018 
of the study was to apply the micronucleus test in erythrocytes of domestic cats (Felis domesticus), and to evaluate them by Giemsa and fluorescent acridine orange (OA) stains carried out sequentially to identify the most suitable for this species. The micronucleus test was applied in peripheral blood erythrocytes of adult domestic cats, with and without signs of disease (total $=24)$. The frequencies of micronuclei detected with OA were 2 to 10 -fold higher than those recorded with Giemsa $(\mathrm{p}<0.0001)$, without differences by age factor or health status. It is hypothesized that $\mathrm{OA}$ staining allowed the detection of micronuclei of smaller size than the Giemsa analysis. The observed frequency of micronuclei proposes the use of the domestic cat to perform biomonitoring on the effect of environmental genotoxics.

Key words: acridine orange; micronucleated erythrocytes; Felis domesticus; pets

\section{INTRODUCCIÓN}

Los contaminantes medioambientales son un reflejo de las características y las actividades propias de cada ambiente, sea este urbano, doméstico, agropecuario o silvestre y, además, pueden ser diferentes de una latitud a otra. En estas situaciones se requiere de la utilización de biomarcadores para evaluar el efecto de los contaminantes sobre animales bioindicadores involucrados en una situación ambiental de riesgo (Capó Martí, 2002). El ambiente urbano se caracteriza por el desarrollo de actividades humanas que liberan una variedad de productos potencialmente tóxicos, de origen industrial, comercial, agrícola o domiciliario. La presencia prolongada de estos contaminantes aumenta el riesgo de exposición de los humanos y animales que conviven con ellos como mascotas, así como de padecer los efectos potenciales producidos por su inhalación, ingestión, o contacto dérmico. Esta situación sugiere que las mascotas puedan ser utilizadas como bioindicadores para realizar bio-monitoreos sobre el efecto de la contaminación ambiental a distintos niveles de organización biológica (Hayes et al., 1991; Reif et al., 1995; Backer et al., 2001).

Los mamíferos son considerados uno de los grupos biológicos más adecuados para ser usados como bioindicadores (Hermoso et al.,
2008). Los científicos William Henry Howell y Justin Marie Jolly encontraron pequeñas inclusiones (remanentes de ADN) en eritrocitos de felinos y ratas, llamadas desde entonces cuerpos de Howell-Jolly, que pueden encontrarse en algunas situaciones patológicas como es el caso de anemias severas (Hayashi, 2016). Estas estructuras luego fueron denominadas micronúcleos $(\mathrm{MN})$, cuya frecuencia también puede ser afectada por factores como especie, edad, sexo, estilo de vida y sensibilidad individual a contaminantes ambientales (Scarfi et al., 1993; Wojda et al., 2007). Los eritrocitos micronucleados (EMN) se forman en el proceso de división celular y son consecuencia de anomalías cromosómicas estructurales y numéricas (Bolognesi y Cirillo, 2014). Los EMN se presentan de manera espontánea en la sangre periférica de algunas especies y su frecuencia se incrementa cuando el individuo se expone a genotóxicos (Zúñiga-González et al., 1998, 2000, 2001).

El análisis de laboratorio para la identificación de los $\mathrm{MN}$ es uno de los más simples y rápidos para biomonitorear los efectos de genotóxicos (Zapata-Restrepo et al., 2017). Los felinos tienen al menos dos condiciones que los hacen particularmente sensibles para ser usados como bioindicadores de ambiente. La molécula de hemoglobina tiene ocho grupos sulfhidrilo comparados con los cuatro grupos de otras especies y, por lo tan- 
to, es más susceptible al estrés oxidativo y a los efectos adversos. Además, los felinos tienen menor capacidad en el bazo, respecto de otras especies, de realizar una remoción de las células dañadas en circulación (Peterson y Talcott, 2006).

Los felinos son mascotas habituales en el hogar, pero también habitan en áreas silvestres, ocupan hábitats extremadamente diversos y están naturalmente distribuidos en todas las regiones zoogeográficas (O'Brien et al., 2008). En el ambiente doméstico, los felinos comparten con los humanos parte de los mismos componentes de la dieta, agua y polvo en suspensión. Una evaluación de los niveles basales de daño citogenético en las poblaciones de animales domésticos es esencial para la implementación e interpretación de bio-monitoreos secuenciales en el tiempo (Udroiu, 2007).

La coloración de Giemsa es la más utilizada para el ensayo de los MN por su sencillez. No obstante, el ensayo de micronúcleos en eritrocitos inmaduros de sangre periférica en ratones con naranja de acridina (NA) fue introducido originalmente por Hayashi y confirmado como equivalente en exactitud al ensayo de MN de médula ósea (Hayashi et al., 1994).

El objetivo de esta investigación fue aplicar el ensayo de micronúcleos en eritrocitos de gatos domésticos adultos, sanos y enfermos, y evaluarlos mediante las coloraciones de Giemsa y naranja de acridina fluorescente realizada en forma secuencial, para identificar la más adecuada para esta especie.

\section{Materiales y Métodos}

\section{Animales}

Se seleccionaron 24 gatos domésticos (Felis domesticus) de una clínica veterinaria de Maipú, Mendoza, Argentina. Los ga- tos eran mestizos, tanto hembras como machos. La mitad eran gatos adultos de 2.5 años en promedio y la otra mitad adultos viejos con 13.5 años promedio. Se consideraron adultos a partir del año, cuando ya han alcanzado su madurez física y sexual; y como adultos viejos a individuos mayores de 10 años (Minovich y Paludi, 2012). Los animales se distribuyeron en cuatro grupos, según grupo etario y estado clínico: adultos y adultos viejos, sanos y con signos clínicos de enfermedad. Se consideraron animales clínicamente sanos a aquellos que al momento de ser evaluados por el profesional veterinario no presentaron signos de enfermedad en el examen clínico ni en los perfiles hematológicos y bioquímicos séricos. Los animales con enfermedad presentaban fallas renales $(\mathrm{n}=3)$, trastornos hepáticos (4), anorexia (2) o hemobartonelosis (3).

Ningún animal recibió medicación durante los 60 días previos a la toma de muestra. Todos los animales recibían alimento balanceado y tenían acceso ad libitum a fuentes de agua potable. Se obtuvo una muestra de sangre periférica de cada ejemplar a partir de la punción de la cara interna del pabellón de la oreja, previa desinfección con alcohol etílico $70^{\circ}$. El protocolo de extracción de muestras fue avalado por el Comité Institucional para el Cuidado y Uso de Animales para Investigación y Docencia (CICUALYD, UMaza).

\section{Coloración Secuencial y Evaluación de las Muestras}

Se utilizaron portaobjetos previamente limpiados con etanol $70^{\circ}$, realizándose dos frotis sanguíneos por cada individuo. Las láminas se fijaron en metanol absoluto durante 10 min y se colorearon con Giemsa (G) (Merck) en una dilución de 1:10 con agua bidestilada, durante $10 \mathrm{~min}$. Se analizaron al microscopio óptico con un aumento de 1000X. Luego fueron decolorados con metanol absoluto y fueron impregnados con $50 \mu 1$ de NA (Basic Orange 14, ®Anedra) $0.003 \%$ en buffer fosfato pH 6.8 (Ueda et al., 1992), con la adaptación de Çavas (Çavas, 2008). 

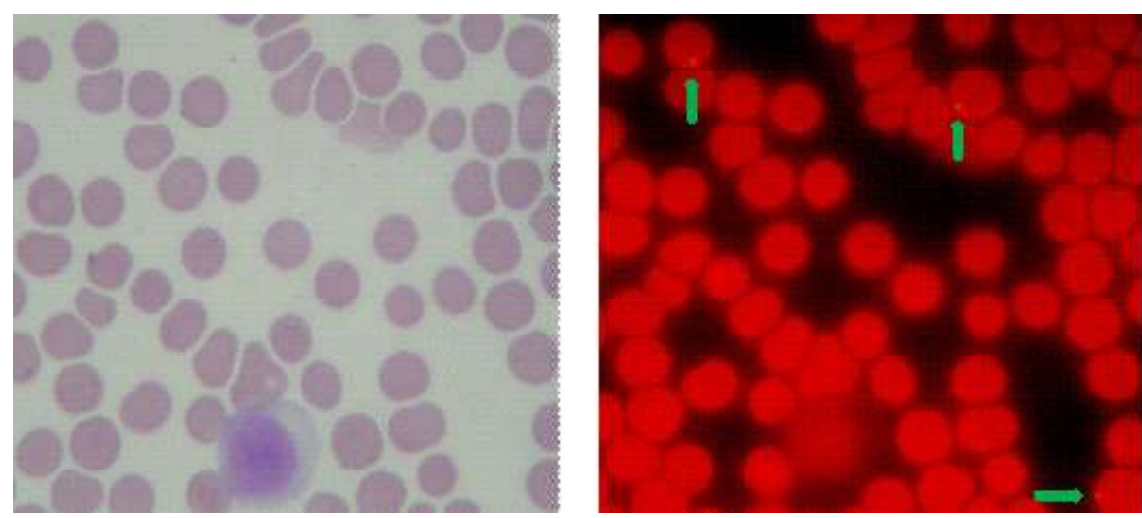

Figura 1. Eritrocitos de gato doméstico con tinción secuencial: Giemsa (izquierda) y naranja de acridina (derecha). Imagen de un mismo campo (1000X). Las flechas indican micronúcleos en la coloración de naranja de acridina, ausentes en la coloración de Giemsa

Los portaobjetos fueron analizados previa codificación. Para cada individuo fueron evaluados 3000 eritrocitos en cada procedimiento de coloración. Fueron analizadas las células que presentaban un citoplasma intacto, sin superposición con otras células, con ausencia de detritus intracitoplasmático y de coloración homogénea. La identificación de los MN se realizó según el criterio de inclusión sugerido inicialmente por Schmid (1975) y revisado por Hayashi et al. (1994). Los resultados fueron expresados como número de células micronucleadas por cada 1000 eritrocitos. Se tomaron fotografías de todos los campos microscópicos analizados donde se encontraron EMN. Se compaginaron las fotos para aparear y comparar el mismo campo celular con las dos coloraciones.

\section{Análisis Estadístico}

Para contrastar la hipótesis de normalidad de los datos obtenidos se aplicó el test de Kolmogorov-Smirnov. Las variables evaluadas fueron: método de coloración, grupo etario y estado de salud. Se comprobó si existían diferencias al nivel de $p<0.05$ entre ambas coloraciones mediante el test de Wilcoxon. A través del test de ANOVA de una vía y postest de Dunnet para cada coloración utilizada se determinó si existieron diferencias entre las frecuencias de EMN según grupo etario y estado sanitario.

\section{Resultados}

Las frecuencias de EMN respondieron a una distribución no paramétrica. En el Cuadro 1 se presentan la frecuencia de eritrocitos micronucleados en los cuatro grupos de gatos domésticos. Se observaron diferencias al nivel de $\mathrm{p}=0.0001$ cuando se compararon los resultados para cada grupo según el método de coloración utilizado, con frecuencias mayores en los análisis realizados con coloración NA. Por otro lado, no se observaron diferencias en la frecuencia de EMN entre los animales sin signo de patologías en comparación con los que presentaron alguna alteración clínica o bioquímica. Tampoco se observaron diferencias entre animales adultos respecto a los adultos viejos.

En la Figura 1 se muestran microfotografías de una muestra con las dos coloraciones realizadas secuencialmente en un mismo campo celular. En esta imagen no se visualizan MN con la coloración de Giemsa 
Cuadro 1. Eritrocitos micronucleados en gatos domésticos analizados con dos coloraciones (seis gatos por grupo)

\begin{tabular}{lcc}
\hline \multirow{2}{*}{ Animales } & \multicolumn{2}{c}{$\begin{array}{c}\text { Número de células micronucleadas/ } 1000 \text { eritrocitos } \\
\text { (media } \pm \text { error estándar) }\end{array}$} \\
\cline { 2 - 3 } & Giemsa & Naranja de acridina \\
\hline Adultos sanos & $4.28 \pm 0.95^{\mathrm{a}}$ & $19.11 \pm 9.16^{\mathrm{b}}$ \\
Adultos enfermos & $5.61 \pm 2.60^{\mathrm{a}}$ & $10.05 \pm 4.33^{\mathrm{b}}$ \\
Adultos viejos sanos & $4.00 \pm 0.53^{\mathrm{a}}$ & $13.44 \pm 2.66^{\mathrm{b}}$ \\
Adultos viejos enfermos & $3.28 \pm 0.85^{\mathrm{a}}$ & $30.33 \pm 11.11^{\mathrm{b}}$ \\
\hline
\end{tabular}

Valores con letras diferentes dentro de filas son estadísticamente diferentes $(p<0.0001)$

mientras que se observan tres EMN con la coloración fluorescente de NA.

\section{Discusión}

Se observó una alta frecuencia de EMN en gatos domésticos adultos respecto al nivel basal de $0.35 \mathrm{EMN}$ por cada 1000 eritrocitos, que es el valor requerido para que una especie sea considerada como buen indicador de la acción de sustancias micronucleogénicas (Zúñiga-González et al., 2000).

Los MN espontáneos no pueden ser observados en la sangre periférica de todas las especies. El sistema retículo endotelial en felinos es deficiente en retirar de la circulación las células dañadas y estas se acumulan en la sangre periférica (Zúñiga-González et al., 2000, 2001) y en número mayor cuando se exponen a genotóxicos ambientales (Zamora-Pérez et al., 2008). Al comparar valores en varias especies de felinos silvestres y el gato doméstico, Zamora-Pérez et al. (2008) encontraron que todos los felinos silvestres, a excepción del león, presentaban valores de EMN más altos que el gato doméstico.
En este estudio, las frecuencias de MN detectadas con naranja de acridina fueron de 2 a 10 veces superiores que las registradas con Giemsa $(p<0.0001)$. En un trabajo previo de este grupo de investigación se encontraron valores con coloración Giemsa de $0.90 \pm$ 0.29 EMN y de $0.76 \pm 0.33$ EMN y con coloración de NA de $1.06 \pm 0.24$ EMN y de 0.86 \pm 0.36 en el gato del pajonal (Leopardus colocolo) y en el gato andino (Leopardus jacobita), respectivamente (Gutiérrez, 2014). Estas frecuencias fueron muy diferentes y más bajas a las obtenidas en el gato doméstico, utilizando el mismo microscopio de fluorescencia y la misma solución colorante. Se hipotetiza que el ambiente silvestre, no urbano y potencialmente exento de contaminantes ambientales podría explicar los niveles más bajos de $\mathrm{MN}$ en los felinos silvestres respecto a los domésticos. Esta observación es opuesta a la descrita por Zamora-Pérez et al. (2008) entre gatos domésticos y silvestres, pero se debe considerar que los ejemplares silvestres analizados por dicho grupo provenían de una colección zoológica inserta en un sitio urbano y no en un ambiente silvestre.

En otras investigaciones en gatos jóvenes, la colchicina y la citosina arabinosa indujeron un $63 \%$ de aumento de los EMN so- 
bre los valores pretratamiento (ZúñigaGonzález et al., 1998), pero con frecuencias por debajo de los observadas en el presente trabajo. Asimismo, se reporta frecuencias de EMN tres veces superiores en gatos jóvenes en comparación a aquellas en gatos adultos (Zúñiga-González et al., 2001). Esas diferencias informadas desde ubicaciones geográficas, edades y coloraciones diferentes confirman la necesidad de establecer valores locales de referencia para cada especie en estudio.

Se ha demostrado que los ensayos son más sensibles cuando se utiliza NA en lugar de Giemsa (Hayashi et al., 1983). En este estudio se observaron artefactos de coloración al utilizar Giemsa. Al emplear portaobjetos coloreados con NA se puede eliminar la presencia de artefactos ambiguos que conducen a falsos positivos (Polard et al., 2011; OECD, 2014). Por otro lado, los mayores niveles de eritrocitos micronucleados con NA observados pueden deberse a la mayor especificidad del fluorocromo por el ácido desoxirribonucleico que permite detectar MN de menor tamaño. En algunas especies de peces, el tamaño pequeño de los cromosomas y por lo tanto de los $\mathrm{MN}$, ha sido señalado para explicar las dificultades encontradas en detectar los MN, de allí que el uso de la coloración fluorescente facilita su detección (Udroiu, 2006). En ratones tratados con ciclofosfamida, que produce $\mathrm{MN}$ muy pequeños, la frecuencia de $\mathrm{MN}$ analizada con Giemsa fue la mitad de la frecuencia obtenida con NA (Tinwell y Ashby, 1989). En mamíferos, la coloración de NA fluorescente ha sido reportada por varios autores como más confiable, permitiendo mayor sensibilidad que Giemsa en células exfoliadas de mucosa bucal humana (Nersesyan et al., 2006).

Los resultados de este estudio indican que el análisis de EMN en gatos domésticos con coloración fluorescente NA es un método que en esta especie puede ser usado para la detección de efecto in situ de agentes genotóxicos.

\section{Agradecimientos}

Los autores agradecen a A. Gutiérrez por la capacitación en la observación por microscopía de fluorescencia; a E. Saldeña por la realización de los montajes fotográficos con coloraciones secuenciales, y a la Universidad Juan Agustín Maza (Mendoza) y al Consejo Nacional de Investigaciones y Técnicas (Argentina) por el soporte económico.

\section{Literatura Citada}

1. Backer LC, Grindem CB, Corbett WT, Cullins L, Hunter JL. 2001. Pet dogs as sentinels for environmental contamination. Sci Total Environ 274: 161-169. doi: 10.1016/S0048-9697(01)00740-9

2. Bolognesi C, Cirillo S. 2014. Genotoxicity biomarkers in aquatic bioindicators. Curr Zool 60: 273-284. doi: 10.1093/czoolo/60.2.273

3. Capó Martí MA. 2002. Principios de ecotoxicología. Diagnóstico, tratamiento y gestión del medio Ambiente. Madrid: McGraw-Hill. $314 \mathrm{p}$.

4. Çavas T. 2008. In vivo genotoxicity of mercury chloride and lead acetate: micronucleus test on acridine orange stained fish cells. Food Chem Toxicol 46: 352-358. doi: 10.1016/j.fct.2007.08.015

5. Gutiérrez AC. 2014. Biomarcadores de daño genético: gato del Pajonal y gato andino como indicadores de calidad en ambientes silvestres. Mendoza: Universidad del Aconcagua. $70 \mathrm{p}$.

6. Hayashi M. 2016. The micronucleus test-most widely used in vivo genotoxicity test. Genes Environ 38. doi: 10.1186/s41021-016-0044-x

7. Hayashi M, Sofuni T, Ishidate M. 1983. An application of acridine orange fluorescent staining to the micronucleus test. Mutat Res 120: 241-247. 
8. Hayashi M, Tice RR, MacGregor JT, Anderson D, Blakey DH, KirshVolders M, Oleson FB, et al. 1994. In vivo rodent erythrocyte micronucleus assay. Mutat Res 312: 293-304.

9. Hayes HM, Tarone RE, Cantor KP, Jessen CR, McCurnin DM, Richardson RC. 1991. Case-control study of canine malignant lymphoma: positive association with dog owner's use of 2,4-dichlorophenoxyacetic acid herbicides. J Natl Cancer Inst 83: 12261231.

10. Hermoso M, García M, Rodríguez FS, López MP. 2008. Los mamíferos salvajes terrestres como bioindicadores: nuevos avances en ecotoxicología. Obs Medioambient 11: 37-62.

11. Minovich, F., Paludi, A. 2012. Medicina felina práctica. Tomo I. $3^{\text {rd }}$ ed. Buenos Aires: Multimédica Ediciones Veterinarias. $398 \mathrm{p}$.

12. Nersesyan A, Kundi M, Atefie $K$, Schulte-Hermann R, Knasmuller $S$. 2006. Effect of staining procedures on the results of micronucleus assays with exfoliated oral mucosa cells. Cancer Epidemiol Biomarkers Prev 15: 18351840. doi: 10.1158/1055-9965.EPI-060248

13. O'Brien SJ, Johnson W, Driscoll C, Pontius J, Pecon-Slattery J, MenottiRaymond M. 2008. State of cat genomics. Trends Genet 24: 268-279. doi: 10.1016/j.tig.2008.03.004

14. OECD [Organisation for Economic Co-operation and Development]. 2014. TG No. 474: Mammalian erythrocyte micronucleus test. [Internet]. Available in: http://www.oecd.org/ publications/test-no-474-mammalianerythrocyte-micronucleus-test9789264224292-en.htm

15. Peterson ME, Talcott PA. 2006. Small animal toxicology. $2^{\text {nd }}$ ed. St. Louis, Missouri: Elsevier. $1232 \mathrm{p}$.

16. Polard T, Jean S, Merlina G, Laplanche C, Pinelli E, Gauthier L. 2011. Giemsa versus acridine orange staining in the fish micronucleus assay and validation for use in water quality monitoring. Ecotoxicol Environ Saf 74: 144-149. doi: 10.1016/j.ecoenv.2010.08.005

17. Reif JS, Lower KS, Ogilvie GK. 1995. Residential exposure to magnetic fields and risk of canine lymphoma. Am J Epidemiol 141:352-359. doi: 10.1093/aje/ 141.4.352

18. Scarfi MR, Lioi MB, Di Berardino D, Zeni O, Coviello AM, Matassino D. 1993. Measurement of micronuclei by cytokinesis-block method in bovine lymphocytes. Mutat Res 289: 291-295. doi: 10.1016/0027-5107(93)90080-Y

19. Schmid W. 1975. The micronucleus test. Mutat Res 31: 9-15. doi: 10.1016/ 0165-1161(75)90058-8

20. Tinwell H, Ashby J. 1989. Comparison of acridine orange and Giemsa stains in several mouse bone marrow micronucleus assays - including a triple dose study. Mutagenesis 4: 476-481. doi: 10.1093/mutage/4.6.476

21. Udroiu I. 2006. The micronucleus test in piscine erythrocytes. Aquat Toxicol 79: 201-204. doi: 10.1016/j.aquatox.2006.06.013

22. Udroiu I. 2007. Cytogenetic monitoring of domestic species. Pak J Biol Sci 10: 831-832.

23. Ueda T, Hayashi M, Ohtsuka Y, Nakamura T, Kobayashi J, Sofuni T. 1992. A preliminary study of the micronucleus test by acridine orange fluorescent staining compared with chromosomal aberration test using fish erythropoietic and embryonic cells. Water Sci Technol 25: 235-240.

24. Wojda A, Zietkiewicz, E, Witt M. 2007. Effects of age and gender on micronucleus and chromosome nondisjunction frequencies in centenarians and younger subjects. Mutagenesis 22: 195-200. doi: 10.1093/mutage/gem002

25. Zamora-Pérez A, Gómez-Meda BC, Ramos-Ibarra ML, Batista-González CM, Luna-Aguirre J, GonzálezRodríguez A, et al. 2008. Los felinos: ¿una alternativa en estudios de 
toxicología genética? Rev Biol Trop 56: 969-974. doi: 10.15517/rbt.v56i2.5638

26. Zapata-Restrepo LM, OrozcoJiménez LY, Rueda-Cardona M, Echavarría SL, Mena-Moreno N, Palacio-Baena JA. 2017. Evaluación genotóxica del agua del Río Grande (Antioquia, Colombia) mediante frecuencia de eritrocitos micronucleados de Brycon henni (Characiformes: Characidae). Rev Biol Trop 65: 405-414. doi: $10.15517 /$ rbt.v65i1.21766

27. Zúñiga-González G, Ramírez-Muñoz MP, Torres-Bugarín O, Pérez-Jiménez J, Ramos-Mora A, Zamora-Pérez A, Gallegos-Arreola MP, et al. 1998. Induction of micronuclei in the domestic cat (Felis domesticus) peripheral blood by colchicine and cytosine-arabinoside. Mutat Res 413: 187-189. doi: 10.1016/ S1383-5718(97)00184-8
28. Zúñiga-González G, Torres-Bugarín O, Zamora-Pérez A, Gómez-Meda BC, Ramos Ibarra ML, MartínezGonzález S, González-Rodríguez A, et al. 2001. Differences in the number of micronucleated erythrocytes among young and adult animals including humans. Spontaneous micronuclei in 43 species. Mutat Res 494: 161-167. doi: 10.1016/S1383-5718(01)00180-2

29. Zúñiga-González G, Torres-Bugarín O, Luna-Aguirre J, GonzálezRodríguez A, Zamora-Pérez A, Gómez-Meda BC, Ventura-Aguilar AJ, et al. 2000. Spontaneous micronuclei in peripheral blood erythrocytes from 54 animal species (mammals, reptiles and birds): part two. Mutat Res 467: 99-103. doi: 10.1016/ S1383-5718(00)00021-8 